\title{
Halophilic Bacterial and Archaeal Communities Composition of the Brine of Tainan Salt Field in Lianyungang, China
}

\author{
Xia Xu*, Zhenya Yuan*, Xiuying Wang, Guozhen Wang, Hongyu Wen*, Dilibaier Paerhati, \\ Qiuyun Luo \\ School of Life Science, Jiangsu Normal University, Xuzhou 221116, Jiangsu, People's Republic of China. \\ *Corresponding Author: Hongyu Wen, School of Life Science, Jiangsu Normal University, No.101, \\ Shanghai road, Tongshan New District, Xuzhou 221116, Jiangsu, People's Republic of China.
}

\begin{abstract}
Brine samples were taken from Tainan Salt Field of Lianyungang, Jiangsu Province, China. After extracting total DNA $16 S$ rRNA gene of the microorganisms, the samples were amplified by PCR and 454 high-throughput sequencing were used for the investigation of bacterial and archaeal communities and species diversity. A total of 6089 bacterial valid reads were obtained from the brine samples and 4589 trimmed reads were remained after trimming. The bacterial community in the sample was dominated by the Acinetobacter (31.36\%) and Delftia (24.10\%) in genus level. A total of 5184 archaeal valid reads were obtained from the brine samples and 4203 trimmed reads were remained after trimming. The archaeal community in the samples was dominated by the Halorubrum (57.77\%) in genus level. The study revealed the community composition of halophilic bacteria and archaea in Tainan Salt Field of Lianyungang, indicating that there are abundant halophilc microorganisms resources and new species in it. Thus, this study could provide theoretical data for the exploration and development of new species in the future.
\end{abstract}

Keywords: halophilic microorganisms; high-throughput sequencing; salt lake; 16S rRNA

\section{INTRODUCTION}

Salt lakes are important mineral resources which contain a variety of salts(mainly $\mathrm{NaCl}$ ). Salt lakes, as a special environment of high salt concentration, are rich in halophilic microorganisms(Oren, 2002).As an important part of the salt lake ecosystem, the halophilic microorganisms have special genetic mechanism and unique metabolites, which have great application research value and development prospect(He \& Lee, 1993). China is a multi-salt lake country, therefore it is of great significance to understand and study the community structure of microorganisms in Chinese salt lakes(Zheng et al., 2002). It is one of the most important tasks for scientific workers to study its genetic evolution, separation and extraction and its comprehensive utilization in combination with the characteristics of Chinese unique and rich mineral resources.

Several researches on the genetics and physiology of microbial resources have focused on highaltitude regions, but studies in low-altitude regions are scanty(Xu et al., 2007). In this study, the extremely halophilic microorganism were cultured from Tainan Salt Field of Lianyungang, Jiangsu Province, China. With further researches done, the use of traditional technologies for environmental microorganisms have been unable to meet the development and utilization of microbial resources(Zhao et al., 2013). Miseq high-throughput sequencing is the second generation of highthroughput sequencing technology, is one of the most widely used Illumina Solexa high-throughput sequencing platform(Huang, 2013; Goodwin et al., 2016), with high accuracy and large throughput, and has been applied in a large number of studies(Guan et al., 2011; Wu et al., 2015; You et al., 2016). This paper reports the results of studies on the diversity of halophilic microorganisms in Tainan Salt Field of Lianyungang using culture, high-throughput sequencing and phylogenetic analysis of selected isolates based on 16S rRNA gene sequences.

\section{MATERIALS AND METHODS}

\section{Site description and sample collection}

The study was carried out in the Tainan Salt Field of Lianyungang, (Longitude 118.4'-119.48',North Latitude 34.7'-35.7'), Jiangsu Province, China, which is an extreme saline environment. The total 
Halophilic Bacterial and Archaeal Communities Composition of the Brine of Tainan Salt Field in Lianyungang, China

hardness of the water is $10 \mathrm{~g} / \mathrm{l}$ and its chemical composition is mainly Chloride, followed by Sodium ion(Table 1). The water samples were collected in the top $10 \mathrm{~cm}$ layer from the salt lake in June 2012. After being sieved through microporous membranes of whose pore size is $0.22 \mu \mathrm{m}$, the samples were stored at $4^{\circ} \mathrm{C}$ before the microbial analysis.

Table 1 General physical and chemical properties for the sampling sites.

\begin{tabular}{|c|c|c|c|c|c|c|}
\hline Sites & $\mathrm{Na}^{+}(\mathrm{mg} / \mathrm{L})$ & $\mathrm{K}^{+}(\mathrm{mg} / \mathrm{L})$ & $\mathrm{Ca}^{2+}(\mathrm{mg} / \mathrm{L})$ & $\mathrm{Cl}^{-}(\mathrm{mg} / \mathrm{L})$ & $\mathrm{SO}_{4}{ }^{2-}(\mathrm{mg} / \mathrm{L})$ & Total hardness $(\mathrm{mg} / \mathrm{L})$ \\
\hline LYG & 53080 & 749 & 878 & 74840 & 7000 & 10000 \\
\hline
\end{tabular}

\section{Strain isolation and culture conditions}

In order to select extremely halophilic microorganisms, we chose high salt medium. The composition of the cultrue medium was as follows: Yeast extract (Oxoid) 10g, Peptone 7.5g, Trisodium citrate 3g, $\mathrm{NaCl} 200 \mathrm{~g}, \mathrm{KCl} 2 \mathrm{~g}, \mathrm{MgSO}_{4} \cdot 7 \mathrm{H}_{2} \mathrm{O} 10 \mathrm{~g}, \mathrm{H}_{2} \mathrm{O} 1000 \mathrm{ml}$, agar powder $20 \mathrm{~g}$ (solid medium), pH 7.5, $121.3^{\circ} \mathrm{C}$, sterilized 25 mins for using later.

Saltern samples were diluted with dilution gradients of $10^{-1}, 10^{-2}, 10^{-3}, 10^{-4}, 10-5$ and $10^{-6}$. Then extracted $100 \mu$ diluted liquid to solid MGM medium plate for a 10 to 15 days spread plate cultivation under the temperature of $37^{\circ} \mathrm{C}$. The cultivated archaea were then streak cultivated to get a single colony. Archaea from the single colony were streak cultivated again to obtain purified strains(Xu et al., 2005; Gerber et al., 1982).

\section{DNA extraction, PCR amplification and high-throughput sequencing}

DNA were extracted according to the instruction of E.Z.N.A Soil DNA gel extraction kit by OMEGA. Details of the procedure will not be listed in this paper.

The 16SrRNA gene sequence of the archaeal strain was amplified by PCR with the following forward and reverse primers: 5'-ACGGGGYGCAGCAGGCGCGA-3' and 5'-GTGCTCCCCCG CCAATTCCT-3'. The PCR condition: $20 \mu 1$ of reaction system, reaction cycles 30 times, $95^{\circ} \mathrm{C}$ predenaturation for $2 \mathrm{~min}, 95^{\circ} \mathrm{C}$ denaturation for $30 \mathrm{~s}, 55^{\circ} \mathrm{C}$ annealing for $30 \mathrm{~s}, 72^{\circ} \mathrm{C}$ extension for $30 \mathrm{~s}$, 30 cycles at $72^{\circ} \mathrm{C}$ for $5 \mathrm{~min}, 10^{\circ} \mathrm{C}$ until halted by uesr.

The 16SrRNA gene sequence of the bacterial strain was amplified by PCR with the following forward and reverse primers: 5'-AGAGTTTGATCCTGGCTCAG-3' and 5'-TTACCGCGGCTGCTGGCAC3'.The PCR condition: $20 \mu 1$ of reaction system,reaction cycles 25 times, $95^{\circ} \mathrm{C}$ predenaturation for $2 \mathrm{~min}, 95^{\circ} \mathrm{C}$ denaturation for $30 \mathrm{~s}, 55^{\circ} \mathrm{C}$ annealing for $30 \mathrm{~s}, 72^{\circ} \mathrm{C}$ extension for $30 \mathrm{~s}, 25$ cycles at $72^{\circ} \mathrm{C}$ for $5 \mathrm{~min}, 10^{\circ} \mathrm{C}$ until halted by uesr.The PCR product was sequenced by Shanghai Majorbio Bio-pharm Technology Co.,Ltd.

According to the preliminary quantitative results of electrophoresis, the PCR products were quantified by QuantiFluor TM -ST Bule Fluorescence Quantitation System. According to the sequencing quantity of each sample, a corresponding proportion of mixed sequencing was followed, followed by Miseq library construction and Miseq sequencing. Sequencing with GS FLX+_ Sequencing Method Manual_XL+Kit.

\section{Analysis of sequence data, and phylogenetic tree construction}

The 16S rRNA gene sequences were compared with the other strains from the EzTaxon Server database. The phylogenetic trees were constructed with the neighbor-joining method of Mega 5.0 software.The topology structure's stability of phylogenetic trees were appraised by using bootstrap value with 1,000 repeats(Felsenstein, 1985).

The 16SrRNA gene sequence of isolated strains have been deposited in the GenBank database and accession numbers were obtained. The similar sequence of the other strains were obtained from the database of EzTaxon Server.

\section{Results}

\section{Phylogenetic analysis of 16S rRNA sequence}

We isolated and enriched 16 strains from the samples, 6 of bacteria , 10 of archaea respectively. After amplifying of PCR, there're 1400bp to $1500 \mathrm{bp}$ lengths of each sample's $16 \mathrm{~S}$ rDNA for phylogenetic 
Halophilic Bacterial and Archaeal Communities Composition of the Brine of Tainan Salt Field in Lianyungang, China

analyses. Two phylogenetic trees were constructed (Fig. 1 and 2), in which Methanosarcina lacustris and Chlamydia abortus were chosen as the out-group. Comparison of the sample sequences with other halobacterial sequences in the database indicated that they were most closely related to the other species which have been reported.

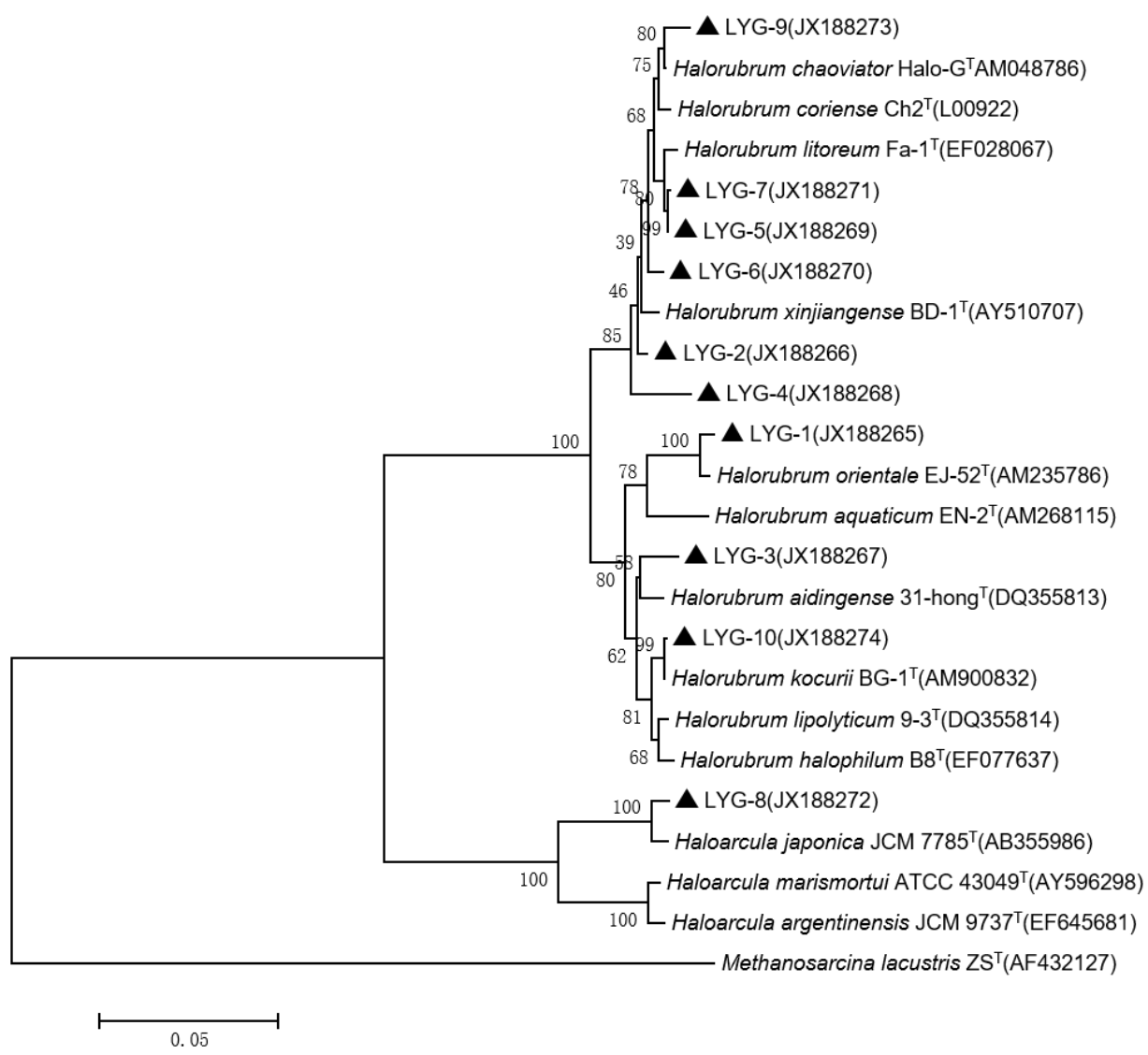

Fig.1 Phylogenetic tree showing the position of LYG halophilic archaeal strains among selected clones or strains belonging to division Halobacteriaceae.The tree, which was rooted using Methanosarcina lacustris as the out-group, was generated by the neighbour-joining method. The number at each branch of phylogenetic tree represents the bootstrap value (1000 replicates).

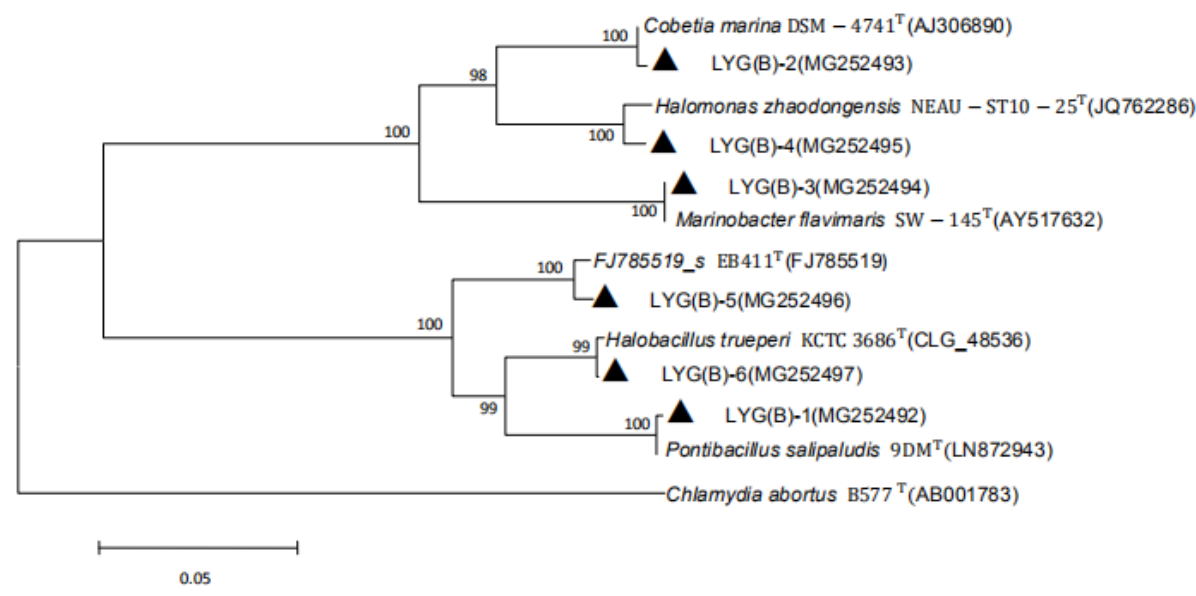

Fig.2 Phylogenetic tree showing the position of LYG halophilic bacterial strains among selected clones or strains belonging to division Halobacteriaceae.The tree, which was rooted using Chlamydia abortus as the outgroup, was generated by the neighbour-joining method. The number at each branch of phylogenetic tree represents the bootstrap value (1000 replicates).

The archaea had 10 different strains and nine of them belonged to genus halorubrum. LYG-1 was clustered into one group with Halorubrum orientale. LYG-4 showed 96\% homology with 
Halophilic Bacterial and Archaeal Communities Composition of the Brine of Tainan Salt Field in Lianyungang, China

Halorubrum xinjiangense, which may represent further members of this novel phylum that is yet to be proposed. LYG-8 was distinct from others that it's close to Haloarcula japonica. Meanwhile,the bacterial species are more classified, each belonging to a different genus.Among them, $\mathrm{LYG}(\mathrm{B})-5$ belonged to genus Lentibacillus.

Archaeal community composition structure

The high-throughput analysis of archaeal 16S rRNA genes resulted in recovery of 5184 effective sequences and obtained 4203 optimized sequences, accounting for $81.08 \%$ of effective sequences, with an average read length $486 \mathrm{bp}$.

Sequences of the samples from the Tainan Salt Field, 360 sequences were unknown or have not been classified as genera $(9.63 \%)$, indicating that there were many potential new species to be discovered. The rest of it mainly dominated by the following 4 genus: Halobellus(7.84\%), Halonotius(10.64\%), Haloplanus(5.32\%), Halorubrum (57.77\%) and the rare genus had 329 sequences, accounting for $8.80 \%$ of all(Table 2 and Fig. 3).

Table 2 The percentages and numbers of sequences in different genera of archaea

\begin{tabular}{|l|l|l|}
\hline Genera & sequence number & Percentage (\%) \\
\hline Halonotius & 398 & 10.64 \\
\hline Haloplanus & 199 & 5.32 \\
\hline Halobellus & 293 & 7.84 \\
\hline Halorubrum & 2160 & 57.77 \\
\hline Others & 329 & 8.80 \\
\hline No_Rank & 360 & 9.63 \\
\hline
\end{tabular}

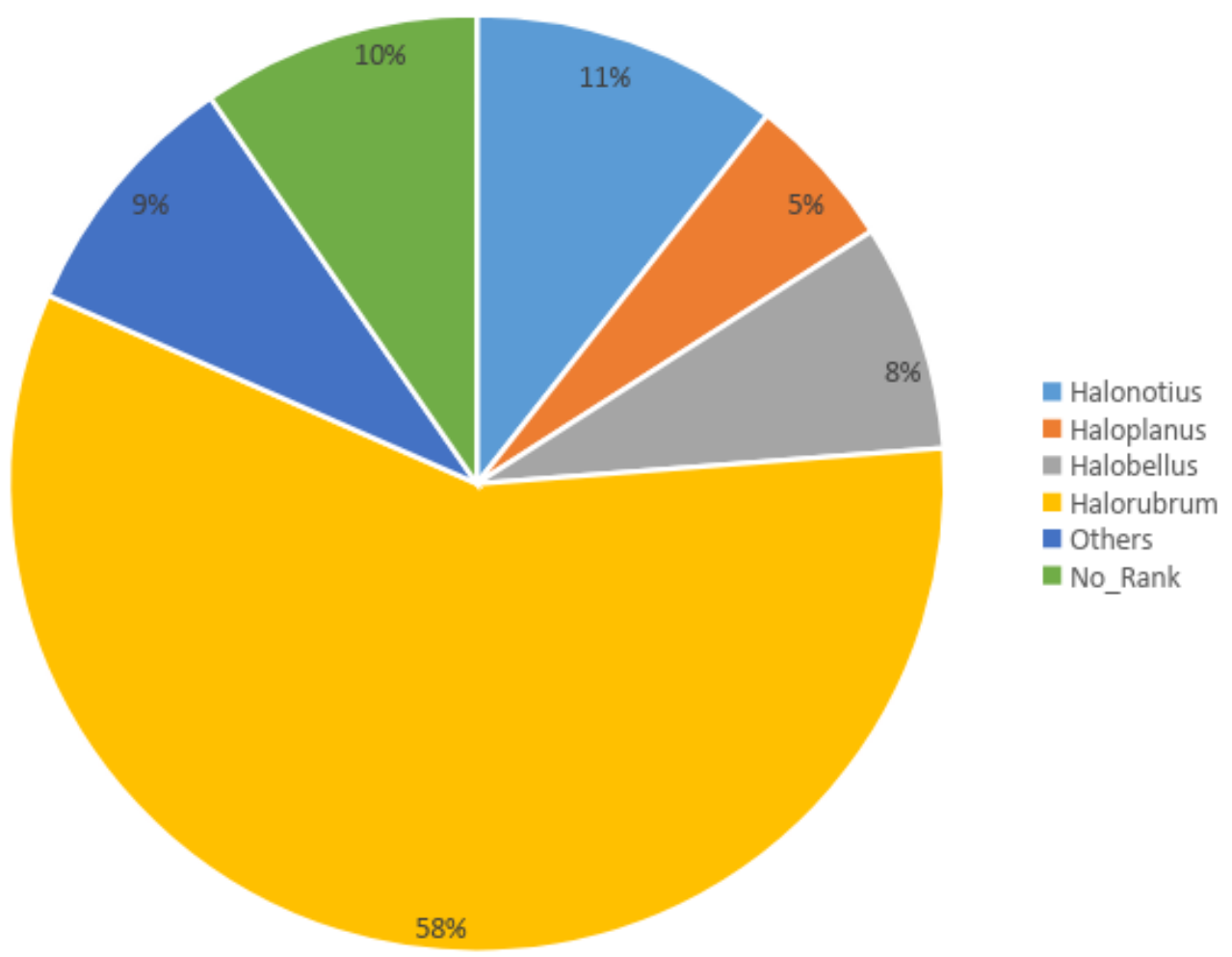

Fig 3. Distribution statistics of the archaeal community at the level of the genus

\section{Bacterial community composition structure}

The high-throughput analysis of bacterial 16S rRNA genes resulted in recovery of 6089 effective sequences and obtained 4589 optimized sequences, accounting for $75.37 \%$ of effective sequences, with an average read length $482 \mathrm{bp}$.

Sequences of the samples from the Tainan salt field, 567 sequences were unknown or have not been classified as genera(12.64\%), indicating that there were many potential new species to be discovered. 
Halophilic Bacterial and Archaeal Communities Composition of the Brine of Tainan Salt Field in Lianyungang, China

The rest of it mainly belonged to the following genus: Acinetobacter (31.36\%), Delftia (24.10\%), Ectothiorhodospiraceae uncultured (3.86\%), Methyloversatilis (4.23\%), Rhizobium (4.08\%), Salinibacter $(10.41 \%)$ and the rare genus had 418 sequences,accounting for $9.32 \%$ of all(Table 3 and Fig. 4).

Table 3 The percentages and numbers of sequences in different genera of bacteria

\begin{tabular}{|l|l|l|}
\hline Genera & sequence number & Percentage (\%) \\
\hline Acinetobacter & 1407 & 31.36 \\
\hline Delftia & 1081 & 24.10 \\
\hline Ectothiorhodospiraceae_uncultured & 173 & 3.86 \\
\hline Methyloversatilis & 190 & 4.23 \\
\hline Rhizobium & 183 & 4.08 \\
\hline Salinibacter & 467 & 10.41 \\
\hline Others & 418 & 9.32 \\
\hline No_Rank & 567 & 12.64 \\
\hline
\end{tabular}

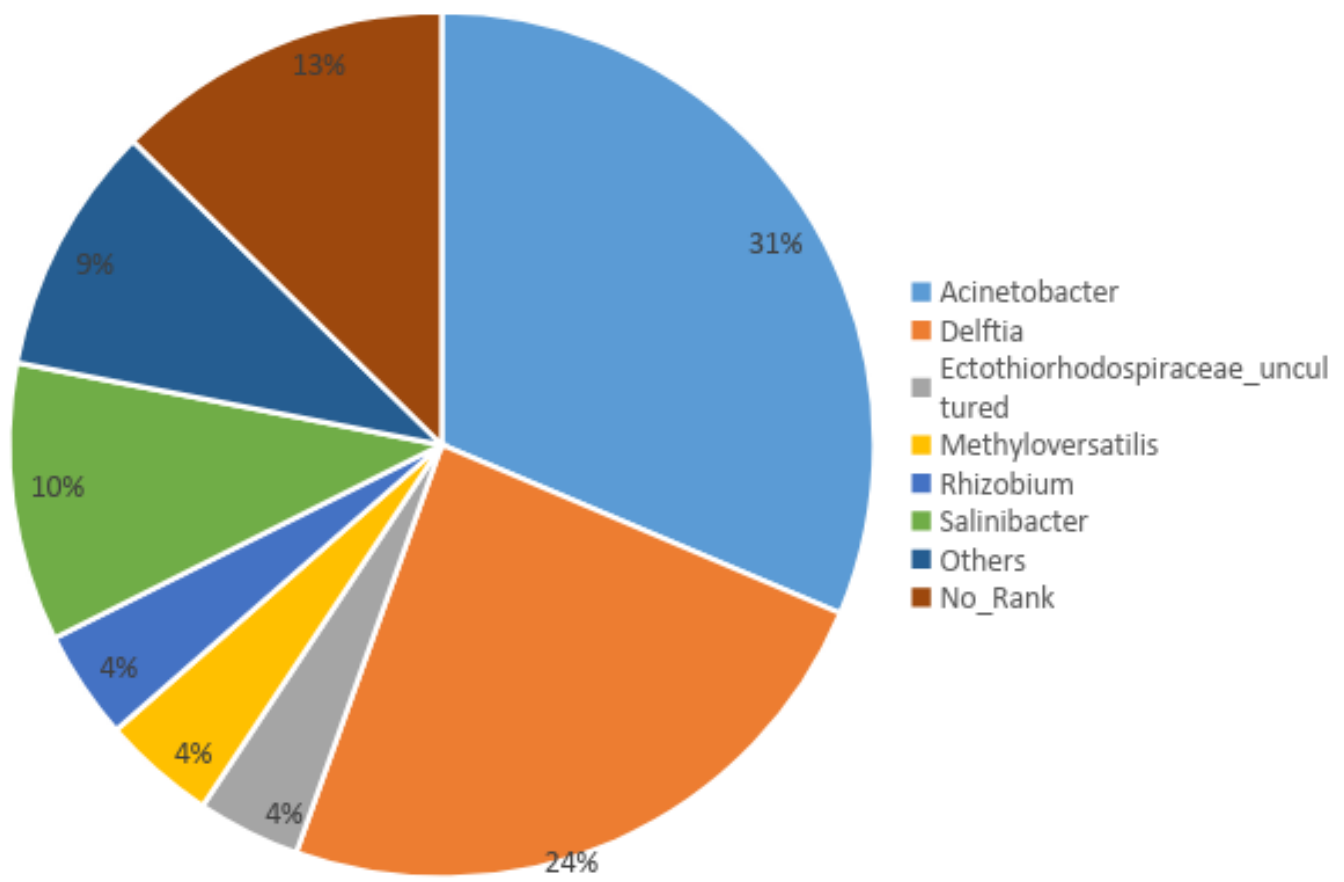

Fig 4. Distribution statistics of the bacterial community at the level of the genus

\section{DISCUSSION}

Lianyungang is located in the South-East Coastal region, having monsoon climate of medium latitudes throughout the year, which can bring warm wind and abundant rainfall(Zheng, 2002). The 10 archaeal strains collected from Tainan salt field demonstrated that sea water was good to breed extremely halophilic archaea. Several years ago, we researched the diversity of extremely halophilic archaea of another salt lake in Lianyungang, and the result showed that the dominant genus was Halorubrum as well(Wen et al., 2009). It illustrated that these areas in Lianyungang have the same natural conditions, and they have a similar effect on extremely halophilic archaea. According to the reference, if the 16S rRNA gene sequences of microbial homology is less than 98\%, they belong to different species. LYG-4 showed 96\% homology with Halorubrum xinjiangense that indicated it may be a novel species had not been found yet. So further investigation is necessary to identify more new microbial species.In contrast, the classification of bacteria is much clear.All of their 16S rRNA gene sequences of microbial homology is more than $98 \%$. Although they belong to different genus, but their species can be determined, as shown by the phylogenetic tree. In summary, bacteria are more abundant. Many kinds of bacteria can adapt to this extreme environment. Nevertheless, archaea are 
Halophilic Bacterial and Archaeal Communities Composition of the Brine of Tainan Salt Field in Lianyungang, China

more specific. Compared with bacteria, less archaeal effective sequences were detected and Halorubrum is predominant.

In this paper, a large number of sequences were obtained by high-throughput sequencing, and the bacterial and archaeal community structures in Tainan Salt Field of Lianyungang were comprehensively analyzed, which could provide some theoretical basis for the comparison of the bacterial and archeael community diversity and species distribution characteristics in the future, and also could provide an important reference to Chinese coastal salt environment microbial species resources and the future development of it.

\section{REFERENCES}

Oren A (2002). Halophilic Microorganisms and their Environments. Kluwer Academic Publisher 5(2): 151-152. He RF, Lee YQ (1993). Progress in the study of halophilic bacteria. Marine Science Bulletin 1993(5): 84-88.

Zheng XY, Zhang MG, Xu C, Lee BX (2002). Summarization of salt lake in China. Science Press,Beijing.

Xu XW, Wu M, Wu YH, Zhang HB (2007). Culturable halophilic archaeal diversity of Ayakekumu salt lake located in Xinjiang, China. Acta Ecol Sinica 27: 3119-3123.

Zhao WY, Yang J, Dong HL, Geng WU, Wang S (2013). Microbial Diversity in the Hypersaline Dabuxun Lake in Qaidam Basin,China. Earth \& Environment41(4): 398-405.

Huang Y (2013). Microbial Genomics Research Based on High Throughput Sequencing. Academy of Military Medical Sciences, Beijing 2013: 9-10.

Goodwin S, McPherson JD, McCombie WR (2016). Coming of age: ten years of next-generation sequencing technologies. Nature Reviews Genetics 17(6): 333.

Guan L, Cho KH, Lee JH (2011). Analysis of the cultivable bacterial community in jeotgal, a Korean salted and fermented seafood, and identification of its dominant bacteria. Food Microbiol 28(1): 101-113.

Wu LY, Wen CQ, Qin YJ (2015). Phasing amplicon sequencing on Illumina Miseq for robust environmental microbial community analysis. BMC Microbiol 15: 125.

You J, Wu G, Ren FP (2016). Microbial community dynamics in Baolige oilfield during MEOR treatment, revealed by Illumina MiSeq sequencing. Environ Biotechnol 100:1469-1478.

Xu XW, Wu M, Huang WD (2005). Isolation and characterization of a novel strain of Natrinema containing a bop gene. Journal of Zhejiang University Science 6B(2): 142-146.

Gerber GE, Anderegg RJ, Herlihy WC (1982). Partial primary structure of bacteriorhodopsin: sequencing methods for membrane proteins. Methods in Enzymology 88: 56-74.

Felsenstein J (1985). Contidence limits on phylogenies: an approach using the bootstrap. The Society for the Study of Evolution 39: 783-791.

Zheng XY (2002). Chinese Salt lakes. Sci Press.

Wen HY, Yang L, Shen LL (2009). Isolation and characterization of culturable halophilic microorganisms of salt ponds in Lianyungang, China. World J Microbiol Biotechnol 25: 1727-1732.

Citation: Xia Xu et al., "Halophilic Bacterial and Archaeal Communities Composition of the Brine of Tainan Salt Field in Lianyungang, China ", International Journal of Research studies in Microbiology and Biotechnology, vol. 4, no. 1, p. 17-22, 2018. http://dx.doi.org/10.20431/2454-9428.0401003

Copyright: () 2018 Authors. This is an open-access article distributed under the terms of the Creative Commons Attribution License, which permits unrestricted use, distribution, and reproduction in any medium, provided the original author and source are credited. 